1 Fundação Oswaldo Cruz (Fiocruz), Centro de Pesquisas Aggeu Magalhães (CPqAM) Recife (PE), Brasil.

nandocastim@yahoo.com.br

2 Fundação Oswaldo Cruz (Fiocruz), Centro de Pesquisas Aggeu Magalhães (CPqAM) Recife (PE), Brasil. paulette.cavalcanti@gmail.com

3 Fundação Oswaldo Cruz (Fiocruz), Centro de Pesquisas Aggeu Magalhães (CPqAM) Recife (PE), Brasil. wayner@cpqam.fiocruz.br

\section{A Estratégia Saúde da Família no estado de Pernambuco: avaliação da estrutura das equipes por porte populacional}

\author{
The Family Health Strategy in the State of Pernambuco: evaluation of \\ team structure by population size
}

Fernando Castim Pimentel1, Paulette Cavalcanti de Albuquerque ${ }^{\mathbf{2}}$, Wayner Vieira de Souza $\mathbf{3}$
RESUMO O estudo teve como objetivo avaliar a estrutura das Equipes de Saúde da Família do estado de Pernambuco por porte populacional, no ano de 2009. Foram aplicados questionários estruturados a 154 equipes, de 24 municípios, e avaliados itens referentes a instrumentos, materiais educativos, espaço físico, medicamentos, vacinas, além dos recursos humanos disponíveis. Os resultados mostraram que os espaços físicos das equipes foram mais bem avaliados nos municípios de pequeno porte $(\mathrm{p}=0,032)$. Somente $5,6 \%$ das equipes cumprem todos os requisitos de estrutura, e o componente recursos humanos apontou a precariedade do vínculo dos profissionais, principalmente nos municípios de médio porte do estado.

PALAVRAS-CHAVE Atenção Primária à Saúde; Estratégia Saúde da Família; Estrutura dos serviços; Recursos humanos em saúde; Avaliação de serviços de saúde.

ABSTRACT The study aimed to evaluate the structure of Health Family Teams of the state of Pernambuco by population size in the year of 2009. Were applied structured questionnaires to 154 teams, from 24 counties, and evaluated items related to instruments, educational materials, physical space, medicines, vaccines, besides the human resources available. The results showed that the physical spaces of the teams were better evaluated in small-sized municipalities $(p=0,032)$. Only 5,6\% of the teams meet all the structure requirements, and the component human resources pointed out the precariousness of the professional bonds, mainly in mid-sized municipalities of the state.

KEYWORDS Primary Health Care; Family Health Strategy; Structure of services; Health manpower; Health services evaluation. 


\section{Introdução}

Com o objetivo de estruturar a assistência à saúde no Brasil, o Programa Saúde da Família (PSF) foi originado em 1994, a partir do Programa de Agentes Comunitários de Saúde (Pacs) (BRASIL, 2006A), e hoje se constitui em estratégia para a organização da Atenção Básica (AB), visando romper com o modelo de atenção centrado na lógica biomédica que privilegia a assistência hospitalar (SILVEIRA FILHO, 2008). Pretendia-se que a implantação da Estratégia Saúde da Família (ESF) reorientasse a forma de atuação das equipes e a organização dos serviços (OLIVEIRA; SALIBA, 2005), consolidando-a nos pequenos e médios municípios e ampliando nos grandes centros urbanos (BRASIL, 2006B).

Em 28 de março de 2006, foi publicada a Política Nacional de Atenção Básica (PNAB), regulamentada pela Portaria n. ${ }^{\circ}$ 648, que visava reforçar os objetivos da ESF, quais sejam: possibilitar o acesso universal e contínuo a serviços de saúde; efetivar a integralidade, seja através da articulação das ações de promoção à saúde, prevenção de agravos, vigilância à saúde, tratamento e reabilitação ou pelo trabalho interdisciplinar e em equipe; valorizar os profissionais de saúde por meio do estímulo e do acompanhamento constante de sua formação e capacitação, entre outros (BRASIL, 2006A). Além disso, a portaria traz os princípios e diretrizes gerais da $\mathrm{AB}$, as responsabilidades dos níveis de gestão, as atribuições dos profissionais e os critérios para implantação e credenciamento das equipes. Atualmente, a ESF é regulada pela portaria n. ${ }^{\circ} 2488$, de 21 de outubro de 2011 (BRASIL, 2011).

Contudo, apesar da normatização estabelecida, a implantação da ESF está na dependência de processos políticos, institucionais, técnicos e organizativos, próprios de cada município, o que gera grande diversidade no seu funcionamento (DENIS; CHAMPAGNE, 1997), principalmente, quanto à estrutura das unidades, vínculo e qualificação dos recursos humanos, bem como o processo de trabalho das equipes. Acredita-se que a maior adesão à ESF ainda ocorra nos municípios de pequeno e médio porte (UCHOA ET AL., 2011), entretanto, a sua implantação não ocorre da mesma forma nos municípios de diferentes portes populacionais (HENRIQUE; CALVO, 2009).

Muitas dificuldades enfrentadas para a implantação das equipes e a manutenção das condições de trabalho adequadas são decorrência da má distribuição dos recursos financeiros, influenciada pelo poder político e porte populacional dos municípios. Investimentos, como o Programa de Expansão e Consolidação da Saúde da Família (Proesf), destinado aos municípios com população acima de 100 mil habitantes, possibilitaram que estes expandissem a sua rede. No caso dos municípios de menor porte populacional e de menor Índice de Desenvolvimento Humano (IDH), essa expansão ocorreu devido ao maior valor per capita de recursos transferidos do Ministério da Saúde para a AB, superior ao dos municípios de grande porte, conforme a Portaria n. ${ }^{\circ}$ 650/GM, de 28 de março de 2006 (BRASIL, 2006C).

Alguns desses problemas foram evidenciados por Tanaka (2011), segundo o qual, a implantação da ESF em municípios de pequeno porte encontrou, inicialmente, facilidades operacionais, embora, em médio prazo, tenha apresentado problemas quanto à fixação do profissional médico; e, nos municípios de grande porte, houve a dificuldade de adscrição da clientela e o frágil reconhecimento como porta de entrada do sistema de saúde. Além disso, outros estudos (VIANA ET AL., 2008; PINTO; MENEZES; VILLA, 2010) constataram a infraestrutura precária das unidades de saúde, com problemas que vão desde a falta de unidades de saúde próprias às condições de apoio diagnóstico e terapêutico (equipamentos, medicamentos e insumos básicos).

A temática da avaliação tem sido bastante utilizada para estudar esses e outros aspectos relacionados à ESF. Uma metodologia 
muito útil, seja para tomada de decisão ou para pesquisa científica, é a avaliação de qualidade, proposta por Donabedian (1988), cujos componentes básicos são: Estrutura, Processo e Resultado, sendo a estrutura, foco deste trabalho, o componente que representa as características relativamente estáveis, como os recursos materiais, humanos e organizacionais. Esse enfoque facilita e permite a melhor sistematização do processo de avaliação (SAMICO ET AL., 2010), além de apresentar uma boa compatibilidade com os programas e serviços de saúde (AGUILAR; ANDER-EGG, 1994).

Starfield (2002) propôs um modelo para avaliar os serviços de Atenção Primária à Saúde (APS) que valoriza a estrutura como um dos componentes para análise do sistema de serviços de saúde. A estrutura corresponde aos recursos necessários para oferecer os serviços, entre eles, a análise de pessoal, que considera a educação e o treinamento de todos os envolvidos na prestação de serviços, e a análise da instalação e dos equipamentos, que inclui o prédio e os componentes físicos da instalação.

Assim, considerando a estrutura um aspecto relevante na assistência à saúde da população, que pode variar de acordo com o porte populacional do município, este artigo tem como objetivo avaliar a estrutura das Equipes de Saúde da Família (EqSF) do estado de Pernambuco, por porte populacional, no ano de 2009.

\section{Metodologia}

Trata-se de um estudo descritivo de corte transversal, no qual foi analisada a estrutura das EqSF do estado de Pernambuco, através da utilização do banco de dados da pesquisa 'Atenção Primária à Saúde no estado de Pernambuco: avaliação do período 19972006 segundo modelo de gestão dos municípios', realizada no ano de 2009, financiada pelo Conselho Nacional de Desenvolvimento Científico e Tecnológico (CNPq), Edital
MCT/CNPq Universal n. ${ }^{\circ}$. 15/2007, faixa B e aprovada pelo Comitê de Ética em Pesquisa do CPqAM/Fiocruz n. ${ }^{\circ}$ 129/2008, em 04 de dezembro de 2008.

O estado de Pernambuco está dividido em 185 municípios. Segundo estimativas censitárias do Instituto Brasileiro de Geografia e Estatística (IBGE), para 2009, ano de realização das entrevistas, Pernambuco tem uma população de 8.810 .318 habitantes, sendo que $89,2 \%$ são cobertos pela APS, entendida aqui como o serviço desenvolvido pelas Unidades de Saúde da Família (USF) ou Unidades Básicas de Saúde (UBS) que possuem população cadastrada e acompanhada pelos Agentes Comunitários de Saúde (ACS) a elas vinculados.

Segundo o Cadastro Nacional de Estabelecimentos de Saúde (CNES), do Departamento de Informação do Ministério da Saúde (Datasus), o estado contava com 1.848 EqSF, à época da realização da pesquisa.

Dos 185 municípios, apenas 10 possuem mais de 100 mil habitantes e tiveram acesso a recursos para na estruturação da ESF, através do Proesf, podendo desenvolver mecanismos de capacitação dos profissionais de forma autônoma, de melhoria das condições de trabalho das unidades, compra de equipamentos etc. Os demais municípios contam apenas com os recursos repassados para o fundo municipal de saúde, a título do Piso da Atenção Básica ou dos incentivos para EqSF, ACS ou Equipes de Saúde Bucal (EqSB).

A população do estudo foi composta pelas EqSF do estado de Pernambuco. Uma amostra foi calculada para estimar uma proporção de $90 \%$ (valor esperado para o prontuário familiar), com erro absoluto de $5 \%$ e grau de confiança de $95 \%$, obtendo-se, assim, 129 equipes. Considerando uma perda amostral de $15 \%$, a amostra final totalizou 152 equipes. Durante a realização da pesquisa, as EqSF foram sorteadas aleatoriamente, respeitando-se a proporção de equipes por porte populacional e por município, o que resultou na aplicação de 154 questionários, 
em 24 municípios do estado. Assim, deveriam ser aplicados 55 questionários nos municípios de grande porte, 69 nos municípios de médio e 30 naqueles de pequeno porte.

Foi selecionado um informante-chave de cada equipe para responder ao questionário, devido, em muitos casos, à dificuldade de paralisar o turno de atendimento da equipe. Entretanto, foi necessário que esse informante apresentasse alguns critérios, como ser integrante da equipe há, pelo menos, um ano e conhecer as atividades desenvolvidas, garantindo, assim, uma maior qualidade das informações.

Foi aplicado um questionário piloto, inicialmente, em uma equipe selecionada intencionalmente, pela facilidade de contato e disponibilidade das equipes em respondê-lo. O teste piloto é de grande importância na detecção de inconsistências e ajustes no instrumento de coleta. A adaptação, a correção e a identificação de alguns aspectos relativos à estrutura oferecida às equipes favoreceram uma avaliação mais fidedigna do modelo de atenção desenvolvido pelas EqSF.

Utilizaram-se questionários estruturados, que, posteriormente, foram digitados no programa Epi Info versão 3.5.3, formando-se, então, um banco de dados com informações sobre as EqSF participantes.

Para efeitos deste trabalho, as variáveis foram divididas em dois componentes: estrutura e recursos humanos. O componente estrutura é composto pelos eixos: a) instrumentos e equipamentos; b) materiais educativos; c) condições dos espaços físicos das unidades; d) vacinas; e) medicamentos essenciais. O componente recursos humanos subdivide-se em: a) composição da equipe; b) capacitação/educação em saúde; c) vínculo dos profissionais.

No eixo referente aos instrumentos e equipamentos, verificou-se a presença dos seguintes itens: estetoscópio, tensiômetro, otoscópio, oftalmoscópio, termômetro, lanterna clínica, foco, aparelho aerossol/nebulizador, balança adulto, balança infantil, material para pequenas cirurgias, material para retirar pontos, glicosímetro, estetoscópio de pinard, sonar dopller, geladeira para vacina, régua antropométrica, caixa térmica ou isopor, mesa ginecológica e outras. No eixo dos materiais educativos, verificou-se a disponibilidade de cartazes e folhetos, álbum seriado, lápis pilot, pincéis, tintas, TV, DVD, vídeos e outros. Nos espaços da unidade, foram observadas a existência e as condições da sala de procedimentos básicos, sala de curativo exclusiva, sala de vacina exclusiva, sala para guarda e dispensação de medicamento, consultório para assistência clínica, consultório equipado para exame Papanicolau, banheiro para usuário, banheiro para funcionário, local para reunião e copa. Com relação às vacinas, observou-se a presença de BCG, antipólio, tríplice/ DTP, tríplice viral/MMR, toxóide tetânico, tetravalente (DTP+Hib), anti-hepatite B, DT Adulto, rotavirus e outras. Por fim, sobre os medicamentos essenciais, analisou-se a disponibilidade de antibióticos, antianêmicos, contraceptivos, anti-hipertensivos, vitamina A, antitérmicos, analgésicos, anti-inflamatórios, broncodilatadores, antiparasitários, antieméticos, hipoglicemiantes orais, antimicóticos, soro reidratante e insulina.

$\mathrm{O}$ eixo relativo aos recursos humanos abordou a composição da equipe mínima, a capacitação e a educação em saúde, como treinamento introdutório ao saúde da família, curso de aperfeiçoamento, especialização, residência, mestrado ou doutorado em saúde pública, treinamento nas áreas AIDPI (Atenção Integrada às Doenças Prevalentes na Infância), diabetes, hipertensão, DST/ Aids, hanseníase, tuberculose, saúde do trabalhador e saúde da mulher. Também foi abordada a forma de vínculo dos profissionais das equipes.

Para a análise dos dados por porte populacional, foram utilizadas informações demográficas. Para isso, foram coletadas informações do IBGE referentes à população residente no ano de estudo. Para este estudo, 
considerou-se município de pequeno porte aquele com menos de 20 mil habitantes; médio porte, o município que possui entre 20 mil e 100 mil habitantes; e o município com mais de 100 mil habitantes foi considerado de grande porte.

Para se analisar as variáveis do estudo com o porte populacional, levou-se em consideração que a distribuição dos questionários aplicados nos municípios foi baseada na proporção de EqSF existentes em Pernambuco, por porte populacional. Entretanto, ao final da coleta dos dados, devido às limitações técnicas e financeiras para visitas a alguns municípios, observou-se uma diferença entre os percentuais de questionários que deveriam ser aplicados e aqueles realmente aplicados. Com isso, a fim de corrigir esse percentual, foi calculado um fator de ponderação, dividindo-se, para cada porte populacional, a proporção de questionários calculados pela proporção de questionários aplicados (tabela 1). Esse fator foi de grande importância na apresentação dos resultados, a fim de que o total fosse expresso com a devida correção.

Tabela 1. Distribuição das Equipes de Saúde da Família dos municípios do estado de Pernambuco, Brasil (agosto de 2008), por porte populacional, segundo questionários aplicados e fator de correção

\begin{tabular}{|c|c|c|c|c|c|c|}
\hline \multirow[t]{2}{*}{ Porte populacional dos municípios } & \multicolumn{2}{|c|}{ ESF em PE } & \multirow{2}{*}{$\begin{array}{r}\begin{array}{r}\text { Questionários } \\
\text { previstos }\end{array} \\
\mathbf{N}\end{array}$} & \multicolumn{2}{|c|}{$\begin{array}{r}\text { Questionários } \\
\text { aplicados }\end{array}$} & \multirow[t]{2}{*}{$\begin{array}{r}\text { Fator de } \\
\text { ponderação }\end{array}$} \\
\hline & $\mathbf{N}$ & $\%$ & & $\mathbf{N}$ & $\%$ & \\
\hline Grande & 652 & 35,3 & 55 & 71 & 46,1 & 0,77 \\
\hline Médio & 832 & 45,0 & 69 & 55 & 35,7 & 1,26 \\
\hline Pequeno & 364 & 19,7 & 30 & 28 & 18,2 & 1,08 \\
\hline Total & 1848 & 100,0 & 154 & 154 & 100,0 & \\
\hline
\end{tabular}

Os resultados obtidos foram representados na forma de frequências e percentuais, em planilhas eletrônicas do software Microsoft Excel (Windows). A análise dos dados também permitiu verificar diferenças entre a estrutura disponível pelas equipes e o porte populacional dos municípios. Para isso, utilizou-se o teste Qui-quadrado e o teste exato de Fisher para comparar as proporções entre esses três estratos, utilizando o programa Epi Info, versão 6.04.

\section{Resultados}

A análise dos equipamentos básicos disponíveis para as EqSF evidenciou a ausência de alguns materiais em muitas equipes, como a lanternaclínica(31,4\%), ooftalmoscópio(7,5\%) e material para pequenas cirurgias $(20,4 \%)$.
$\mathrm{Na}$ análise de distribuição por porte, alguns equipamentos apresentaram-se com uma menor frequência em municípios de pequeno porte (otoscópio e régua antropométrica), o foco mostrou-se em menor quantidade nos municípios de médio porte e a balança infantil nos municípios de grande porte, todos com diferença estatisticamente significativa (tabela 2).

Com relação aos materiais educativos, constatou-se que cartazes/folhetos $(94,6 \%)$ e álbum seriado (87\%) estiveram presentes na maior parte das equipes (tabela 2). Entretanto, materiais lúdicos, como lápis piloto, pincéis e tintas $(57,6 \%)$, além de materiais de maior custo, como TV, DVD e vídeos (26,3\%), demonstraram uma menor disponibilidade pelas equipes. Percebe-se, também, a realidade de cada município ao se comparar que a disponibilidade de recursos mais modernos 
(como TV,DVD e vídeos) esteve mais relacionada aos municípios de grande porte $(\mathrm{p}=0,000)$, enquanto os recursos de menor custo (como lápis, pincéis e tintas) mostraram-se mais frequentes nos municípios de pequeno porte, sem diferença significativa $(\mathrm{p}=0,069)$.

Tabela 2. Distribuição das Equipes de Saúde da Família dos municípios do estado de Pernambuco por porte populacional, segundo instrumentos, materiais educativos e espaço físico, 2009

\begin{tabular}{|c|c|c|c|c|c|c|c|c|c|}
\hline \multirow{2}{*}{ INSTRUMENTOS/ EQUIPAMENTOS } & \multicolumn{2}{|c|}{ TOTAL (154) } & \multicolumn{2}{|c|}{ GP (71) } & \multicolumn{2}{|c|}{ MP (55) } & \multicolumn{2}{|c|}{ PP (28) } & \multirow{2}{*}{ p-value } \\
\hline & $\mathbf{N}$ & $\%$ & $\mathbf{N}$ & $\%$ & $\mathbf{N}$ & $\%$ & $\mathbf{N}$ & $\%$ & \\
\hline Estetoscópio & 152,5 & 99,0 & 69 & 97,2 & 55 & 100,0 & 28 & 100,0 & 0,668 \\
\hline Tensiômetro & 153,2 & 99,5 & 70 & 98,6 & 55 & 100,0 & 28 & 100,0 & 1,000 \\
\hline Otoscópio & 106,9 & 69,4 & 57 & 80,3 & 39 & 70,9 & 13 & 46,4 & 0,004 \\
\hline Oftalmoscópio & 11,5 & 7,5 & 7 & 9,9 & 4 & 7,3 & 1 & 3,6 & 0,566 \\
\hline Termômetro & 150,2 & 97,5 & 71 & 100,0 & 52 & 94,5 & 28 & 100,0 & 0,084 \\
\hline Lanterna clínica & 48,3 & 31,4 & 21 & 29,6 & 17 & 30,9 & 10 & 35,7 & 0,837 \\
\hline Foco & 128,8 & 83,6 & 64 & 90,1 & 41 & 74,5 & 26 & 92,9 & 0,023 \\
\hline Aparelho aerossol/nebulizador & 138,9 & 90,2 & 59 & 83,1 & 52 & 94,5 & 26 & 92,9 & 0,097 \\
\hline Balança adulto & 148,4 & 96,4 & 67 & 94,4 & 53 & 96,4 & 28 & 100,0 & 0,424 \\
\hline Balança infantil & 145,7 & 94,6 & 63 & 88,7 & 55 & 100,0 & 26 & 92,9 & 0,039 \\
\hline Material de pequenas cirurgias & 31,4 & 20,4 & 13 & 18,3 & 11 & 20,0 & 7 & 25,0 & 0,756 \\
\hline Material para retirar pontos & 137,2 & 89,1 & 62 & 87,3 & 48 & 87,3 & 27 & 96,4 & 0,378 \\
\hline Glicosímetro & 147,1 & 95,5 & 65 & 91,5 & 54 & 98,2 & 27 & 96,4 & 0,229 \\
\hline Estetoscópio de Pinard & 95,6 & 62,1 & 51 & 71,8 & 32 & 58,2 & 15 & 53,6 & 0,136 \\
\hline Sonar Dopller & 131,9 & 85,6 & 60 & 84,5 & 45 & 81,8 & 27 & 96,4 & 0,183 \\
\hline Geladeira para vacina & 149,8 & 97,3 & 70 & 98,6 & 54 & 98,2 & 26 & 92,9 & 0,242 \\
\hline Régua antropométrica & 144,0 & 93,5 & 70 & 98,6 & 52 & 94,5 & 23 & 82,1 & 0,007 \\
\hline Caixa térmica ou isopor & 150,6 & 97,8 & 71 & 100,0 & 54 & 98,2 & 26 & 92,9 & 0,040 \\
\hline Mesa ginecológica & 137,5 & 89,3 & 61 & 85,9 & 48 & 87,3 & 28 & 100,0 & 0,116 \\
\hline Outras & 9,6 & 6,3 & 6 & 8,5 & 4 & 7,3 & 0 & 0,0 & 0,294 \\
\hline \multicolumn{10}{|l|}{ MATERIAIS EDUCATIVOS } \\
\hline Cartazes e folhetos & 144,8 & 94,6 & 67 & 95,7 & 51 & 92,7 & 27 & 96,4 & 0,689 \\
\hline Álbum seriado & 133,2 & 87,0 & 61 & 87,1 & 48 & 87,3 & 24 & 85,7 & 0,978 \\
\hline Lápis pilot, pincéis, tintas & 88,1 & 57,6 & 44 & 62,9 & 26 & 47,3 & 20 & 71,4 & 0,069 \\
\hline TV, DVD e vídeos & 40,2 & 26,3 & 33 & 47,1 & 5 & 9,1 & 8 & 28,6 & 0,000 \\
\hline \multicolumn{10}{|l|}{ ESPAC̣OS DA UNIDADE } \\
\hline Sala de procedimentos básicos & 86,0 & 55,9 & 30 & 42,6 & 32 & 58,0 & 21 & 76,7 & 0,009 \\
\hline Sala de curativo exclusiva & 74,3 & 48,2 & 25 & 35,2 & 30 & 55,1 & 16 & 56,7 & 0,042 \\
\hline Sala de vacina exclusiva & 115,5 & 75,5 & 49 & 70,0 & 43 & 78,3 & 22 & 80,0 & 0,501 \\
\hline $\begin{array}{l}\text { Sala para guarda e dispensação de } \\
\text { medicamento }\end{array}$ & 101,8 & 66,1 & 45 & 63,0 & 38 & 69,6 & 18 & 63,3 & 0,789 \\
\hline Consultório para atend. clínico & 123,9 & 80,4 & 54 & 75,9 & 44 & 79,7 & 25 & 90,0 & 0,335 \\
\hline $\begin{array}{l}\text { Consultório equipado para exame } \\
\text { Papanicolau }\end{array}$ & 100,2 & 65,1 & 49 & 68,5 & 30 & 55,1 & 23 & 83,3 & 0,034 \\
\hline Banheiro para usuário & 106,4 & 69,1 & 47 & 66,7 & 37 & 68,1 & 22 & 80,0 & 0,465 \\
\hline Banheiro para funcionário & 103,4 & 67,1 & 47 & 66,7 & 38 & 69,6 & 18 & 63,3 & 0,895 \\
\hline Local para reunião (máx 2 km) & 74,1 & 48,1 & 30 & 42,6 & 26 & 46,4 & 17 & 60,0 & 0,253 \\
\hline Copa & 81,5 & 52,9 & 37 & 51,9 & 31 & 56,5 & 13 & 46,7 & 0,688 \\
\hline
\end{tabular}

Fonte: Elaboração própria

$\mathrm{GP}=$ Grande Porte, $\mathrm{MP}=$ Médio Porte, $\mathrm{PP}=$ Pequeno Porte

OBS 1: Os totais apresentados ( $\mathrm{N} \mathrm{e} \mathrm{\% )} \mathrm{estão} \mathrm{corrigidos} \mathrm{pelo} \mathrm{fator} \mathrm{de} \mathrm{ponderação}$

OBS 2: Quando houve valores perdidos (missing), os mesmos foram considerados no cálculo dos percentuais 
A respeito da estrutura física, as equipes foram questionadas sobre a existência e as condições dos espaços nas unidades. Observa-se, na tabela 2, que a maior parte dos espaços estava presente e em boas condições de uso. Porém, a sala de curativo exclusiva (48,2\%), o local para reunião (48,1\%), a copa $(52,9 \%)$ e a sala de procedimentos básicos $(55,9 \%)$ foram áreas das unidades citadas com menor frequência pelas equipes.

Considerando as vacinas (tabela 3 ) como elementos essenciais às ações básicas de prevenção de doenças, nota-se que existem poucas unidades com déficits de vacinas importantes. A vacina menos citada como disponível em quantidade suficiente foi o toxóide tetânico (80,5\%). Com relação à distribuição por porte, não se observou diferença significativa entre os municípios. Vacinas como a antirrábica e a dupla viral, apesar de não constarem no questionário, foram relatadas pelos profissionais das equipes e, assim, registradas no item 'outras'.

Tabela 3. Distribuição das Equipes de Saúde da Família dos municípios do estado de Pernambuco por porte populacional, segundo disponibilidade de vacinas e medicamentos essenciais, 2009

\begin{tabular}{|c|c|c|c|c|c|c|c|c|c|}
\hline \multirow{2}{*}{ VACINAS } & \multicolumn{2}{|c|}{ TOTAL (153) } & \multicolumn{2}{|c|}{ GP (71) } & \multicolumn{2}{|c|}{ MP (55) } & \multicolumn{2}{|c|}{ PP (28) } & \multirow{2}{*}{ p-value } \\
\hline & $\mathbf{N}$ & $\%$ & $\mathbf{N}$ & $\%$ & $\mathrm{~N}$ & $\%$ & $\mathbf{N}$ & $\%$ & \\
\hline$B C G$ & 149,0 & 96,8 & 69 & 97,2 & 54 & 98,2 & 26 & 92,9 & 0,339 \\
\hline Antipólio & 154,0 & 100,0 & 71 & 100,0 & 55 & 100,0 & 28 & 100,0 & 1,000 \\
\hline Tríplice/DTP & 152,7 & 99,2 & 71 & 100,0 & 54 & 98,2 & 28 & 100,0 & 0,539 \\
\hline Tríplice Viral/MMR & 152,9 & 99,3 & 71 & 100,0 & 55 & 100,0 & 27 & 96,4 & 0,182 \\
\hline Toxóide Tetânico & 124,0 & 80,5 & 53 & 74,6 & 49 & 89,1 & 20 & 71,4 & 0,075 \\
\hline Tetravalente (DTP+Hib) & 150,2 & 97,5 & 71 & 100,0 & 52 & 94,5 & 28 & 100,0 & 0,084 \\
\hline Anti-Hepatite B & 154,0 & 100,0 & 71 & 100,0 & 55 & 100,0 & 28 & 100,0 & 1,000 \\
\hline DT Adulto & 152,7 & 100,0 & 71 & 100,0 & 54 & 100,0 & 28 & 100,0 & 1,000 \\
\hline Rotavírus & 150,9 & 98,0 & 70 & 98,6 & 54 & 98,2 & 27 & 96,4 & 0,771 \\
\hline Outras & 39,8 & 25,9 & 16 & 22,5 & 15 & 27,3 & 8 & 28,6 & 0,753 \\
\hline \multicolumn{10}{|c|}{ MEDICAMENTOS ESSENCIAIS } \\
\hline Antibióticos & 98,8 & 64,6 & 46 & 65,7 & 35 & 63,6 & 18 & 64,3 & 0,970 \\
\hline Antianêmicos & 124,6 & 81,4 & 50 & 71,4 & 47 & 85,5 & 25 & 89,3 & 0,058 \\
\hline Contraceptivos & 130,3 & 85,2 & 53 & 75,7 & 48 & 87,3 & 27 & 96,4 & 0,028 \\
\hline Anti-hipertensivos & 113,2 & 74,0 & 41 & 58,6 & 46 & 83,6 & 22 & 78,6 & 0,006 \\
\hline Vitamina A & 120,3 & 78,6 & 47 & 67,1 & 48 & 87,3 & 22 & 78,6 & 0,030 \\
\hline Antitérmicos & 131,3 & 85,8 & 54 & 77,1 & 49 & 89,1 & 26 & 92,9 & 0,074 \\
\hline Analgésicos & 125,4 & 81,9 & 51 & 72,9 & 47 & 85,5 & 25 & 89,3 & 0,090 \\
\hline Anti-inflamatórios & 98,1 & 64,1 & 42 & 60,0 & 36 & 65,5 & 19 & 67,9 & 0,709 \\
\hline Broncodilatadores & 102,6 & 67,1 & 50 & 71,4 & 33 & 60,0 & 21 & 75,0 & 0,269 \\
\hline Antiparasitários & 120,6 & 78,8 & 53 & 75,7 & 42 & 76,4 & 25 & 89,3 & 0,302 \\
\hline Antieméticos & 108,8 & 71,1 & 47 & 67,1 & 38 & 69,1 & 23 & 82,1 & 0,323 \\
\hline Hipoglicemiantes orais & 122,5 & 80,1 & 48 & 68,6 & 50 & 90,9 & 21 & 75,0 & 0,011 \\
\hline Antimicóticos & 96,2 & 64,1 & 44 & 65,7 & 35 & 63,6 & 17 & 60,7 & 0,898 \\
\hline Soro reidratante & 129,2 & 84,4 & 56 & 80,0 & 47 & 85,5 & 25 & 89,3 & 0,481 \\
\hline Insulina & 30,4 & 20,0 & 10 & 14,5 & 12 & 21,8 & 7 & 25,0 & 0,398 \\
\hline
\end{tabular}

Fonte: Elaboração própria

$\mathrm{GP}=$ Grande Porte, $\mathrm{MP}=$ Médio Porte, $\mathrm{PP}=$ Pequeno Porte

OBS 1: Os totais apresentados ( $\mathrm{Ne} \%$ ) estão corrigidos pelo fator de ponderação

OBS 2: Quando houve valores perdidos (missing), os mesmos foram considerados no cálculo dos percentuais 
Também foi possível constatar que determinados medicamentos, os quais não poderiam faltar nas USF, não estavam disponíveis ou encontravam-se em quantidade insuficiente para a demanda da equipe, como, por exemplo, antibióticos, anti-inflamatórios e broncodilatadores, presentes em quantidade suficiente em apenas $64,6 \%, 64,1 \%$ e $67,1 \%$ das equipes, respectivamente (tabela 3 ). Vale ressaltar que alguns medicamentos, como contraceptivos, anti-hipertensivos, vitamina A e hipoglicemiantes orais, foram encontrados em menor frequência nos municípios de grande porte quando comparados com os municípios de médio e pequeno porte.

A tabela 4 mostra um consolidado dos eixos - equipamentos, materiais educativos, espaços da unidade, vacinas e medicamentos essenciais - apresentados anteriormente nas tabelas 2 e 3. Pode-se constatar que a maioria das equipes apresentou disponibilidade mínima de dois materiais educativos (89,6\%) e presença de todas as vacinas necessárias para a população (74,3\%). Entretanto, 17,5\% das equipes demonstraram dificuldades em disponibilizar o quantitativo mínimo de materiais/instrumentos/equipamentos, e $32,9 \%$ possuíam os medicamentos essenciais em quantidade suficiente. Com relação aos espaços físicos, $55,7 \%$ das equipes afirmaram estarem em boas condições de uso, sendo essa condição mais frequente nos municípios de pequeno porte, quando comparada à daqueles de grande porte $(\mathrm{p}=0,032)$.

Constatou-se, ainda na tabela 4 , que $61,7 \%$ das equipes cumpriram, pelo menos, 3 dos 5 eixos (equipamentos, materiais educativos, espaço físico, vacinas e medicamentos), sendo as equipes dos municípios de pequeno porte as que apresentaram um maior percentual de cumprimento, comparadas com as equipes dos municípios de grande porte $(\mathrm{p}=0,000)$. Ao se avaliar o cumprimento de todos os eixos do componente estrutura, apenas 5,6\% das EqSF de Pernambuco cumpriram $100 \%$, sem diferença estatística significativa.

Tabela 4. Distribuição das Equipes de Saúde da Família do estado de Pernambuco segundo critérios avaliativos do componente estrutura, por porte populacional, 2009

\begin{tabular}{|c|c|c|c|c|c|c|c|c|c|}
\hline \multirow{2}{*}{ COMPONENTE ESTRUTURA } & \multicolumn{2}{|c|}{ TOTAL (154) } & \multicolumn{2}{|c|}{ GP (71) } & \multicolumn{2}{|c|}{ MP (55) } & \multicolumn{2}{|c|}{ PP (28) } & \multirow{2}{*}{$\mathrm{p}$-value } \\
\hline & $\mathbf{N}$ & $\%$ & $\mathbf{N}$ & $\%$ & $\mathbf{N}$ & $\%$ & $\mathbf{N}$ & $\%$ & \\
\hline $\begin{array}{l}\text { Equipes que possuem, pelo menos, } 90 \% \text { dos } \\
\text { instrumentos/ equipamentos }\end{array}$ & 27,0 & 17,5 & 15 & 21,1 & 8 & 14,5 & 5 & 17,9 & 0,636 \\
\hline $\begin{array}{l}\text { Equipes que possuem, no mínimo, } 2 \text { materiais } \\
\text { educativos }\end{array}$ & 137,9 & 89,6 & 66 & 93,0 & 47 & 85,5 & 26 & 92,9 & 0,325 \\
\hline $\begin{array}{l}\text { Equipes que possuem, pelo menos, } 70 \% \text { da estrutura } \\
\text { física em boas condições de uso }\end{array}$ & 85,7 & 55,7 & 31 & 43,7 & 32 & 58,2 & 20 & 71,4 & 0,032 \\
\hline $\begin{array}{l}\text { Equipes que possuem } 100 \% \text { das vacinas em } \\
\text { quantidade suficiente }\end{array}$ & 114,5 & 74,3 & 50 & 70,4 & 45 & 81,8 & 18 & 64,3 & 0,173 \\
\hline $\begin{array}{l}\text { Equipes que possuem, pelo menos, } 90 \% \\
\text { dos medicamentos essenciais em quantidade suficiente }\end{array}$ & 50,7 & 32,9 & 26 & 36,6 & 15 & 27,3 & 11 & 39,3 & 0,432 \\
\hline Equipes que cumprem 3 dos critérios acima & 95,0 & 61,7 & 29 & 40,8 & 38 & 69,1 & 23 & 82,1 & 0,000 \\
\hline Equipes que cumprem todos os critérios acima & 8,6 & 5,6 & 2 & 2,8 & 3 & 5,5 & 3 & 10,7 & 0,279 \\
\hline
\end{tabular}

Fonte: Elaboração própria

$\mathrm{GP}=$ Grande Porte, $\mathrm{MP}=$ Médio Porte, $\mathrm{PP}=$ Pequeno Porte

OBS 1: Os totais apresentados ( $\mathrm{N}$ e \%) estão corrigidos pelo fator de ponderação

OBS 2: Quando houve valores perdidos (missing), os mesmos foram considerados no cálculo dos percentuais 
$\mathrm{Na}$ análise do componente relativo aos recursos humanos (tabela 5), observou-se que $83,6 \%$ das equipes apresentavam-se completas, com composição mínima de 1 médico, 1 enfermeiro, 1 técnico ou auxiliar de enfermagem e 4 a 12 ACS. Com relação à capacitação/educação em saúde dos profissionais, constatou-se que $63,9 \%$ dos profissionais que participaram da pesquisa possuíam treinamento introdutório em saúde da família, $53,8 \%$ possuíam algum curso na área de saúde pública/saúde da família e $73,9 \%$ possuíam treinamento em, pelo menos, 4 das áreas citadas. Ressalta-se que o percentual de equipes com treinamento introdutório foi maior nos municípios de grande porte, quando comparadas às dos municípios de médio e pequeno porte $(\mathrm{p}=0,002)$. $O$ percentual de equipes com cursos na área de saúde pública/saúde da família e treinamento nas áreas básicas também foi maior nos municípios de grande porte, no entanto, sem demonstrar diferença estatística significativa.

Com relação ao vínculo dos profissionais, constatou-se que apenas $24,1 \%$ dos profissionais entrevistados foi por concurso público, principalmente nos municípios de grande porte, comparados aos de médio porte $(\mathrm{p}=0,001)$.

Por fim, observou-se que $65,6 \%$ das equipes de Pernambuco apresentaram cumprimento de, pelo menos, 3 dos 5 eixos do componente recursos humanos, e que apenas $5,4 \%$ das equipes cumpriam todos os eixos desse componente (tabela 5). Em ambos os casos, os municípios de grande porte apresentaram melhores resultados quando comparados aos municípios de médio e pequeno porte.

Tabela 5. Distribuição das Equipes de Saúde da Família do estado de Pernambuco segundo critérios avaliativos do componente recursos humanos, por porte populacional, 2009

\begin{tabular}{|c|c|c|c|c|c|c|c|c|c|}
\hline \multirow{2}{*}{ COMPONENTE RECURSOS HUMANOS } & \multicolumn{2}{|c|}{ TOTAL (154) } & \multicolumn{2}{|c|}{ GP (71) } & \multicolumn{2}{|c|}{ MP (55) } & \multicolumn{2}{|c|}{ PP (28) } & \multirow{2}{*}{ p-value } \\
\hline & $\mathbf{N}$ & $\%$ & $\mathbf{N}$ & $\%$ & $\mathbf{N}$ & $\%$ & $\mathrm{~N}$ & $\%$ & \\
\hline Equipe mínima completa & 128,8 & 83,6 & 59 & 83,1 & 44 & 80,0 & 26 & 92,9 & 0,317 \\
\hline $\begin{array}{l}\text { Possui treinamento introdutório ao Saúde da } \\
\text { Família? }\end{array}$ & 96,4 & 63,9 & 57 & 81,4 & 29 & 53,7 & 15 & 55,6 & 0,002 \\
\hline $\begin{array}{l}\text { Possui curso de aperfeiçoamento, especialização, } \\
\text { residência, mestrado ou doutorado em saúde } \\
\text { pública? }\end{array}$ & 82,9 & 53,8 & 44 & 62,0 & 27 & 49,1 & 14 & 50,0 & 0,293 \\
\hline Possui treinamento em áreas estratégicas? & 110,8 & 73,9 & 52 & 76,5 & 40 & 74,1 & 19 & 67,9 & 0,858 \\
\hline $\begin{array}{l}\text { Forma de vínculo 'concurso' dos profissionais da } \\
\text { equipe }\end{array}$ & 36,4 & 24,1 & 28 & 41,2 & 5 & 9,1 & 8 & 28,6 & 0,001 \\
\hline Equipes que cumprem 3 dos critérios acima & 101,0 & 65,6 & 58 & 81,7 & 32 & 58,2 & 15 & 53,6 & 0,004 \\
\hline Equipes que cumprem todos os critérios acima & 8,3 & 5,4 & 8 & 11,3 & 0 & 0,0 & 2 & 7,1 & 0,039 \\
\hline
\end{tabular}

Fonte: Elaboração própria

$\mathrm{GP}=$ Grande Porte, $\mathrm{MP}=$ Médio Porte, $\mathrm{PP}=$ Pequeno Porte

OBS 1: Os totais apresentados ( $\mathrm{N}$ e \%) estão corrigidos pelo fator de ponderação

OBS 2: Quando houve valores perdidos (missing), os mesmos foram considerados no cálculo dos percentuais

OBS 3: Considerou-se o mínimo de 4 áreas estratégicas (AIDPI; Diabetes; Hipertensão; DST/Aids; Hanseníase;

Tuberculose; Saúde do Trabalhador; Saúde da Mulher)

\section{Discussão}

\section{Componente estrutura}

A análise da estrutura disponível nas EqSF de Pernambuco demonstrou a presença de instrumentos e equipamentos importantes, como estetoscópio, tensiômetro, termômetro, balança adulto, glicosímetro, geladeira para vacina, entre outros, mas também a ausência de itens importantes para o desenvolvimento do trabalho, como lanterna clínica, 
oftalmoscópio e material para pequenas cirurgias, os quais, segundo o manual de estrutura física das UBS (BRASIL, 2008), são imprescindíveis para o funcionamento de uma UBS com EqSF. O relatório de avaliação da implantação e do funcionamento do PSF, publicado pelo Ministério da Saúde, em 1999 (BRASIL, 1999), constatou a pouca disponibilidade de oftalmoscópio (14,6\%), glicosímetro (29,7\%), sonar doppler (44,9\%), lanterna $(52,2 \%)$, material para pequenas cirurgias $(56,6 \%)$, entre outros, pouco disponíveis até os dias de hoje, como se pode observar no presente estudo.

Outros autores também constataram a pouca disponibilidade desses itens, como Moura et al. (2010), que, ao caracterizarem a modalidade de organização da atenção primária em dois municípios do estado da Bahia, observaram que menos de $50 \%$ das unidades de saúde do município A possuíam tensiômetro infantil, otoscópio, lanterna, oftalmoscópio, materiais para pequenas cirurgias, entre outros. No município B, além dos citados, menos da metade das USF apresentava glicosímetro com fitas e nenhuma possuía oftalmoscópios e equipamentos audiovisuais. Rocha et al. (2012), ao avaliarem o desempenho da ESF no município de Campina Grande (PB), observaram que 90\% das unidades não dispunham nem de oftalmoscópio nem de lanterna; $60 \%, 35 \%$ e $5 \%$ não possuíam negatoscópio, otoscópio e glicosímetro, respectivamente.

Algumas dessas dificuldades também foram encontradas por Pinto, Menezes e Villa, (2010) ao analisarem as situações de trabalho vivenciadas pelos profissionais da ESF do município de Ceará-Mirim (RN), onde $86,8 \%$ dos profissionais indicaram não ter disponibilidade de equipamentos e instrumentos.

A disponibilidade de materiais educativos pelas equipes variou conforme o tipo de material e o porte populacional do município. Os recursos de maior custo (como TV, DVD e vídeos) estavam mais associados aos municípios de grande porte, e os materiais de menor custo mais frequentes nos municípios de pequeno porte, evidenciando a influência do recurso financeiro dos municípios sobre o tipo de atividade educativa desenvolvida. Esses fatores influenciam a qualidade da atenção aos usuários, uma vez que podem dificultar a realização de atividades de promoção à saúde, prevenção de agravos e comprometer a continuidade do cuidado (MOURA ET AL., 2010). Entretanto, pode-se evidenciar que a disponibilidade dos materiais educativos melhorou ao longo dos anos, tendo em vista que, em 1999, a avaliação do PSF (BRASIL, 1999) constatou que apenas $27,3 \%$ das equipes dispunham desse tipo de material em quantidade suficiente.

Com relação aos espaços das unidades, apesar de muitos ambientes terem sido citados com maior frequência, a sala de curativo exclusiva e o local para reunião foram citados por menos da metade das equipes como existentes e em boas condições de uso. Outros autores mostram a disponibilidade de sala de espera, consultórios, cozinha, sala de vacina, farmácia e sala para cuidados de enfermagem, porém, a sala de espera, recepção/arquivo, sala para cuidados de enfermagem e sala de vacinação foram as que tiveram maior quantidade de parâmetros inadequados (ROCHA ET AL., 2012). Também é evidente a ausência de sala de reunião em grande parte das unidades, o que pode dificultar a realização de atividades coletivas com a comunidade e a articulação das práticas de avaliação e planejamento entre os profissionais (MOURA ET AL., 2010).

A existência de vacinas em quantidade suficiente foi outro aspecto verificado nesse estudo, evidenciando boa disponibilidade nas UBS, diferente dos achados da avaliação realizada pelo Ministério da Saúde (BRASIL, 1999), onde vacinas como BCG, anti-hepatite $\mathrm{B}$, toxóide tetânico, tríplice viral e DT infantil eram disponibilizadas por $76,3 \%$, $75,3 \%, 64,6 \%, 64,3 \%$ e $38,3 \%$ das equipes, respectivamente. 
Com relação aos medicamentos essenciais, constatou-se uma menor disponibilidade, visto que apenas $32,9 \%$ das equipes possuíam mais de $90 \%$ dos itens avaliados. O estudo de Rocha et al. (2012) demonstra semelhanças ao constatar que $75 \%$ e $20 \%$ das unidades pesquisadas não dispunham de cobertura vacinal para a BCG e para a dupla viral, respectivamente. Entre os fármacos que deveriam estar disponíveis, os que mais faltaram foram: diclofenaco de potássio (95\%), aminofilina e nistatina creme vaginal (90\%), cimetidina (75\%), neomicina com bacitracina (75\%) e ampicilina (55\%). Em Ceará-Mirim (RN), 83,2\% dos profissionais relataram como principal dificuldade para o desempenho das suas atividades a indisponibilidade de medicamentos (PINTO; MENEZES; VILLA, 2010).

Cabe ressaltar que a Portaria n. ${ }^{\circ} 2.488$ ainda destaca, entre as responsabilidades atribuídas às secretarias municipais de saúde: "garantir recursos materiais, equipamentos e insumos suficientes para o funcionamento das UBS e para a execução do conjunto de ações propostas" (BRASIL, 2011), o que não foi observado neste estudo, principalmente ao se constatar que apenas 5,6\% das EqSF de Pernambuco cumpriram 100\% do componente estrutura, o qual aponta os itens mínimos apresentados pelas equipes para o desenvolvimento adequado das ações de saúde.

\section{Componente recursos humanos}

O componente recursos humanos analisou, inicialmente, a completude das EqSF, e constatou que $16,4 \%$ delas funcionavam sem a composição mínima estabelecida na Portaria n. ${ }^{\circ} 2.488$, de 21 de outubro de 2011 (BRASIL, 2011). Segundo esse documento, a equipe multiprofissional deve ser composta por, no mínimo: médico generalista ou especialista em saúde da família ou médico de família e comunidade, enfermeiro generalista ou especialista em saúde da família, auxiliar ou técnico de enfermagem e ACS, podendo acrescentar a essa composição, como parte da equipe multiprofissional, os profissionais de saúde bucal: cirurgião dentista generalista ou especialista em saúde da família, auxiliar e/ou técnico em saúde bucal.

$\mathrm{Na}$ análise do componente recursos humanos, ficou evidente a relação entre a qualificação dos profissionais e o porte populacional dos municípios, principalmente quando se trata do treinamento introdutório, mais frequente nos municípios de grande porte. Esse achado confirma o maior acesso das equipes de municípios mais desenvolvidos aos cursos de capacitação e ao treinamento em saúde.

Os próprios profissionais reconhecem a importância da equipe com perfil e formação em saúde pública ou em $\mathrm{AB}$, aspecto mencionado por $93,2 \%$ dos profissionais de saúde da família do município de CearáMirim (RN), como facilitador do desenvolvimento das ações em saúde (PINTO; MENEZES; VILLA, 2010). Além disso, outro fator que dificulta a consolidação da ESF está relacionado à formação acadêmica tradicional que esses profissionais receberam durante seus cursos de graduação, baseados em modelos flexnerianos, com currículo rígido e pouco conectado com a realidade da população (SANTOS; CUTOLO, 2003).

A estabilidade dos profissionais através do vínculo foi outro aspecto que se mostrou deficiente, pois apenas $24,1 \%$ dos profissionais que participaram da pesquisa referiram como seu vínculo o concurso público. Esse fato é confirmado por outros estudos que apontam a precariedade de vínculos e a oferta de empregos no setor privado como os principais problemas a serem enfrentados pelos gestores, o que compromete os laços de compromisso e corresponsabilidade entre profissionais de saúde e população (PINTO, 2008).

Diante das dificuldades relativas à qualificação e ao vínculo dos profissionais das EqSF, a Portaria n. ${ }^{\circ} 2.488$ (2011) afirma, em 
seu artigo I, parágrafo VI, que é responsabilidade comum a todas as esferas de governo:

Desenvolver mecanismos técnicos e estratégias organizacionais de qualificação da força de trabalho para gestão e atenção à saúde, valorizar os profissionais de saúde estimulando e viabilizando a formação e educação permanente dos profissionais das equipes, a garantia de direitos trabalhistas e previdenciários, a qualificação dos vínculos de trabalho e a implantação de carreiras que associem desenvolvimento do trabalhador com qualificação dos serviços ofertados aos usuários. (BRASIL, 2011).

Uma das limitações deste estudo refere-se ao seu desenho do tipo transversal, o qual não permite estabelecer relações entre causa e efeito, o que limita a interpretação dos resultados. Outra limitação refere-se ao fato de que as informações utilizadas para se avaliar as equipes eram autorreferidas, não sendo possível, assim, comprovar a veracidade das informações fornecidas, tendo em vista que as informações não eram complementadas pelos pesquisadores através da observação in loco da unidade.

Por fim, alguns autores afirmam que não é possível dizer se os aparatos institucionais de apoio às equipes - como educação permanente e gestão do trabalho, incluindo, aí, vínculos empregatícios - e a infraestrutura das unidades de saúde se diferenciam entre os municípios (SARTI ET AL., 2012).

Entretanto, Henrique e Calvo (2009), avaliando o grau de implantação de municípios catarinenses, demonstraram que os municípios menores apresentaram-se mais bem classificados quanto à estrutura, onde a proporção de municípios com até 20 mil habitantes, nas categorias bom e médio, foi significativamente maior do que a proporção dos demais municípios, o que se assemelha bastante aos resultados encontrados no presente estudo. Esses autores explicam que houve uma tendência inicial de implantação do programa em áreas menos assistidas, caracterizadas por serem municípios de pequeno ou médio porte, onde o incentivo financeiro ao PSF tinha grande poder de indução da política de saúde (HENRIQUE; CALVO, 2009).

Assim, esperava-se que as equipes dos municípios de grande porte apresentassem melhores resultados devido aos investimentos do Proesf, destinados aos municípios com população acima de 100 mil habitantes. Esses municípios apresentaram melhores resultados na avaliação do componente recursos humanos, possivelmente devido ao seu maior acesso à qualificação. Como os municípios de menor porte populacional apresentam maior valor per capita de recursos para a $\mathrm{AB}$, quando comparado ao valor do incentivo dos municípios de grande porte (BRASIL, 2006C), talvez isso explique o melhor desempenho dos municípios de menor porte na avaliação do componente estrutura.

\section{Conclusão}

Constataram-se, através desse estudo, algumas dificuldades vivenciadas pelas EqSF do estado de Pernambuco. A análise do componente estrutura evidenciou que a maioria das equipes dispõe de materiais educativos e vacinas. Entretanto, poucas equipes possuíam a relação dos instrumentos/equipamentos básicos necessários à assistência à saúde da população. Os espaços das unidades mostraram que poucas delas dispõem de local para reunião.

A avaliação geral constatou que pouco mais da metade das equipes cumpre mais de $50 \%$ dos eixos do componente estrutura, com destaque para os municípios de pequeno porte, e apenas $5,6 \%$ cumprem todos os requisitos, o que demonstra a falta de investimento na manutenção das unidades e na aquisição de materiais, insumos, instrumentos e equipamentos, fundamentais para a prestação de uma assistência de qualidade para a população.

Com relação ao componente recursos humanos, os municípios de grande porte 
apresentaram melhores resultados, principalmente quanto ao vínculo dos profissionais e ao treinamento introdutório ao saúde da família.

Dessa forma, é necessário investir na estrutura física das USF dos municípios de grande porte, assim como na qualificação e no reforço do vínculo dos profissionais dos municípios de médio e pequeno porte, a fim de reduzir muitas das diferenças encontradas na ESF do estado de Pernambuco.

\section{Referências}

AGUILAR, M. J.; ANDER-EGG, E. Avaliação de programas e serviços sociais. Petrópolis: Editora Vozes, 1994.

BRASIL. Ministério da Saúde. Avaliação da implantação e funcionamento do Programa de Saúde da Família: relatório preliminar. Brasília, DF, 1999, 72 p.

Ministério da Saúde. Portaria MS/GM, n. ${ }^{\circ} 2488$, de 21 de outubro de 2011. Aprova a Política Nacional de Atenção Básica, para a Estratégia Saúde da Família (ESF) e o Programa de Agentes Comunitários de Saúde (PACS) [internet]. Diário Odicial [da] União. Brasília, DF, 2011. Disponível em: <http://bvsms.saude.gov.br/ bvs/saudelegis/gm/2011/prt2488_21_10_2011.html>. Acesso em: 10 out. 2014.

Ministério da Saúde. Portaria n. ${ }^{\circ}$ 648, de 28 de março de 2006. Aprova a Política Nacional de Atenção Básica, estabelecendo a revisão de diretrizes e normas para a organização da Atenção Básica para o Programa Saúde da Família (PSF) e o Programa Agentes Comunitários de Saúde (PACS). Diário Oficial [da] União Brasília, DF, 2006a.

Ministério da Saúde. Portaria n. ${ }^{\circ}$ 650/GM, de 28 de março de 2006. Define valores de financiamento do PAB fixo e variável mediante a revisão de diretrizes e normas para a organização da Atenção Básica, para a Estratégia Saúde da Família e para o Programa de Agentes Comunitários de Saúde, instituídos pela Política Nacional de Atenção Básica. Diário Oficial [da] União Brasília Brasília, DF, 2006c.

Ministério da Saúde. Secretaria de Atenção à Saúde. Departamento de Atenção Básica. Manual de estrutura física das unidades básicas de saúde: saúde da família. Ministério da Saúde, Secretaria de Atenção à Saúde, Departamento de Atenção Básica - 2. ed. Brasília: Ministério da Saúde, 2008. 52 p.

Ministério da Saúde. Secretaria Executiva, Departamento de Apoio à Descentralização. Diretrizes operacionais dos Pactos pela Vida, em Defesa do SUS e da Gestão. Brasília, DF, 2006b.

DENIS, J.; CHAMPAGNE. F. Análise de implantação. In: HARTZ, Z. M. A. Avaliação em saúde: dos modelos conceituais à prática na análise de implantação de programas. Rio de Janeiro: Ed. Fiocruz, 1997.

DONABEDIAN, A. The quality of care. How can it be assessed? JAMA, Chicago, v. 260, p.1743-1748, 1988.

HENRIQUE, F; CALVO, M. C. M. Grau de implantação do Programa Saúde da Família e indicadores sociais. Ciência e Saúde Coletiva, v. 14, supl.1, Rio de Janeiro, set./out. 2009.

MOURA, B. L. A. et al. Atenção primária à saúde: estrutura das unidades como componente da atenção à saúde. Revista Brasileira de Saúde Materno Infantil, Recife, v. 10 supl. 1, p. 69-81, nov. 2010.

OLIVEIRA, J. L. C.; SALIBA, N. A. Atenção odontológica no Programa de Saúde da Família de Campos dos Goytacazes. Ciência \&t Saúde Coletiva, Rio de Janeiro, v. 10, supl., p. 297-302, 2005.

PINTO, E. S. G.; MENEZES, R.M.P.; VILLA, T.C.S. Situação de trabalho dos profissionais da Estratégia Saúde da Família em Ceará-Mirim. Rev Esc Enferm USP. v. 44, n. 3, p. 657-64, 2010. 
PINTO, E. S. G. Dificuldades e/ou facilidades vivenciadas pelos profissionais que compõem a Estratégia de Saúde da Família. 2008. 119f. Dissertação (Mestrado em Enfermagem) - Departamento de Enfermagem, Universidade Federal do Rio Grande do Norte, Natal, 2008.

ROCHA, A. C. D. et al. Atenção básica à saúde: avaliação de estrutura e processo. RAS, São Paulo, v. 14, n. 54, abr./jun. 2012.

SAMICO, I. et al. (Org.). Abordagens metodológicas na avaliação em saúde. In: Avaliação em Saúde: bases conceituais e operacionais. Rio de Janeiro: Medbook, 2010.

STARFIELD, B. Atenção Primária: equilíbrio entre necessidades de saúde, serviços e tecnologia. Brasília: Unesco/Ministério da Saúde, 2002.

SARTI, T. D. et al. Avaliação das ações de planejamento em saúde empreendidas por equipes de saúde da família. Cadernos de Saúde Pública, Rio de Janeiro, v. 28, n. 3, p. 537-548, 2012.

SANTOS, M. A. M.; CUTOLO, L. R. A. A interdisciplinaridade e o trabalho em equipe no Programa de Saúde da Família. ACM Arquivos Catarinenses de Medicina, Santa Catarina, v. 32, n. 4, p. 65-74, 2003.
SILVEIRA FILHO, A. D. Processo de trabalho das equipes multiprofissionais sob a lógica da Estratégia de Saúde da Família: O uso das ferramentas de Saúde da Família na construção do cuidado em saúde. In: MOYSES, S. T.; KRIGER, L.; MOYSES, S. J. Saúde Bucal das Famílias: trabalhando com evidências. São Paulo: Artes Médicas, 2008.

TANAKA, O. Y. Avaliação da Atenção Básica em Saúde: uma nova proposta. Saúde e Sociedade, São Paulo, v. 20, n. 4, p. 927-934, 2011.

UCHOA, A . C. et al. Avaliação da satisfação do usuário do Programa de Saúde da Família na zona rural de dois pequenos municípios do Rio Grande do Norte. Physis - Revista de Saúde Coletiva, Rio de Janeiro, v. 21, n. 3, p. 1061-1076, 2011.

VIANA, A. L. A. et al. Atenção básica e dinâmica urbana nos grandes municípios paulistas, Brasil. Cadernos de Saúde Pública, Rio de Janeiro, v. 24, supl. 1, p. 79-90, 2008.

Recebido para publicação em março de 2014

Versão final em outubro de 2014

Conflito de interesse: inexistente

Suporte financeiro: Conselho Nacional de Desenvolvimento

Científico e Tecnológico (CNPq), Edital MCT/CNPq Universal

no $15 / 2007$, faixa B 